\title{
Using Geometric Constraints to Solve the Point Correspondence Problem in Fringe Projection Based 3D Measuring Systems
}

\author{
Christian Bräuer-Burchardt, Christoph Munkelt, Matthias Heinze, \\ Peter Kühmstedt, and Gunther Notni
}

\author{
Fraunhofer Institute Applied Optics and Precision Engineering, Jena, Germany \\ \{christian.braeuer-burchardt, christoph.munkelt, matthias.heinze, \\ peter.kuehmstedt, gunther.notni\}@iof.fraunhofer.de
}

\begin{abstract}
A new method for fringe projection based 3D stereo scanners is introduced which realizes point correspondence finding and subsequent unwrapping of phase images without binary codes. The novelty of the method is the combination of geometric constraints between the three optical sensor components together with the estimated measurement accuracy of the system in order to achieve unique point correspondences. Considerable fringe code reduction obtained by use of geometric constraints and Gray code omission leads to a speed-up of the image sequence acquisition time. This opens the possibility to design moving sensors and to measure moving objects.
\end{abstract}

Keywords: fringe projection, phase unwrapping, optical measurement systems, measuring accuracy, epipolar geometry.

\section{Introduction}

Contactless metrology systems based on fringe projection technique are increasingly used for industrial, technical, and medical applications. Although flexibility, measuring accuracy, measurement data volume, and fields of application always increase, processing time should be reduced. Projection of structured light is used in many measuring systems for 3D surface acquisition. Recently considerable progress has been achieved in the field of contactless optical 3D measurements.

An extensive survey over coded structured light techniques to solve the correspondence problem which is the basis for $3 \mathrm{D}$ surface reconstruction is given by Battle et al. [1]. Coded structured light does not only include fringes but also other patterns like barcodes or stochastic patterns. In our work, however, we use exclusively fringe patterns. Methods using projection of sinusoidal fringe patterns have to solve the problem of phase unwrapping. This can be realized e.g. by the use of multiple spatial frequencies [2], temporal phase unwrapping methods [3], or use of Gray code sequences [4]. Due to its unambiguousness, use of Gray code leads to robust results. However, longer image sequences must be recorded, which limits the possible applications. We aim for significant reduction of the number of images of a structured light pattern sequence by omission of the Gray code and to speed-up the image recording time of 
fringe projection 3D measurement systems. This leads to non-static applications and therefore real-time applications or moving objects measurement become possible.

Zhang and Yau suggest a real-time coordinate measurement [5] where phase unwrapping is realized by determination and tracking of a marker. An interesting method for phase unwrapping using at least two cameras is presented by Ishiyama et al. [6]. There the number of possible correspondences is drastically reduced by backpropagation of the correspondence candidates into the image of the second camera. Ishiyama gives another suggestion for $3 \mathrm{D}$ measurement using a one projector one camera fringe projection system [7] using the invariance of cross-ratio of perspective projection. Young et al. [8] suggest the use of the limitation of the measuring volume in order to reduce the search area for corresponding points on the epipolar line to segments achieving a reduction of the projected binary code by careful placement of additional cameras (or additional measuring positions). Li et al. [9] use this approach in combination with the multi-frequency technique in order to realize real-time 3D measurements.

In our recent work we already achieved code reduction by using epipolar constraint and measuring volume restriction (see $[10,11])$.

In this paper, an algorithm is presented which realizes point correspondence finding and phase unwrapping of single frequency fringe patterns without use of the Gray code at all which reduces the length of the projected fringe pattern sequences considerably. This is useful for applications where a short recording time of the image sequences is necessary as for example reconstruction of living objects (e.g. face recognition) and other measurement tasks which require real-time processing.

\section{Model and Approach}

\subsection{Situation and Model}

A number of fringe projection systems for 3D surface determination were developed at our institute $[12,13]$. All of them base on the projection and observation of two orthogonal fringe sequences consisting of Gray code (5 to 7 images) and sinusoidal fringe patterns (between 3 and 16 images). From these sequences phase values are determined using a 4-, 8-, or 16-phase algorithm [13]. Phase values are used together with the extrinsic and intrinsic parameters of the optical components to determine the 3D point coordinates of the reconstructed object surface. Using epipolar constraint in order to find point correspondences is a typical task in phototogrammetry [14]. It reduces the task to a correspondence problem between two one-dimensional vectors.

Using fringe projection the difficult and uncertain task of correspondence finding (CFT) based on intensity correlation may become unambiguous using a robust phase unwrapping method, e.g. Gray code. However, 3D measurement systems based on fringe projection provide more geometric information which can be used for solving the CFT without using Gray code.

Let us consider using a sensor consisting of two cameras $\mathrm{C}_{1}$ and $\mathrm{C}_{2}$ and one projector $\mathrm{P}$ in a fix geometric arrangement (see fig. 1). Intrinsic and extrinsic parameters of all three components are known achieved either by self-calibration using current measurement data [15] or by a-priori calibration. 
Each point $p$ in the camera $\mathrm{C}_{1}$ maps a $3 \mathrm{D}$ point $M$ and corresponds to a straight line $g$ in the image of camera $\mathrm{C}_{2}$. If the measurement volume $m v$ is restricted, the corresponding area of the line $g$ is reduced to a segment $s$ (see fig. 2). Point $q$ corresponding to point $q$ is expected to lie on $s$. A further restriction of the corresponding point candidates is achieved by the phase value obtained from the projected fringe pattern sequence $[13,15]$. Since we want to omit the Gray code sequence which would make the CFT unique we use the rough phase values occurring periodically with a period length depending on the projected pattern.

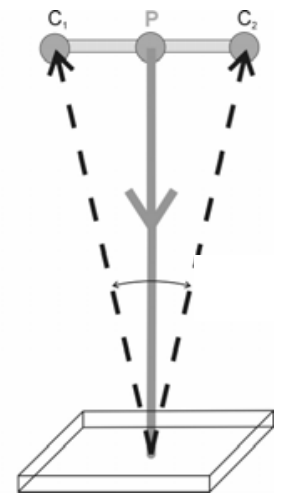

Fig. 1. Sensor arrangement

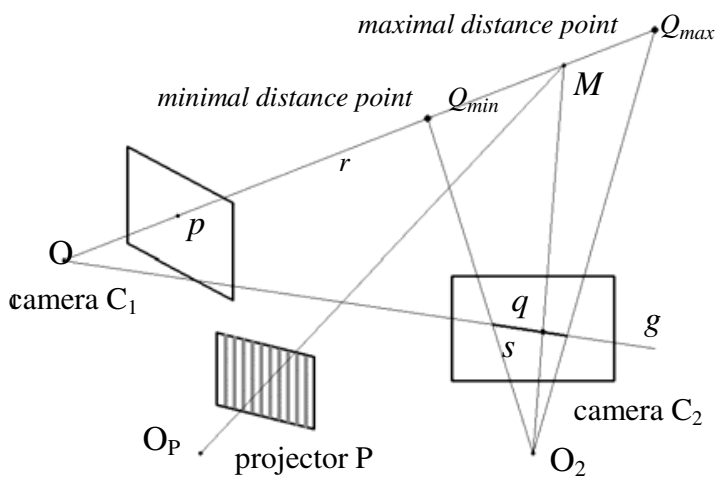

Fig. 2. Epipolar geometry with segment $s$

We typically use a projection display with an active area of 1024 x 1024 pixels, a period length of 16 pixels according to 64 fringes in maximum over the measurement volume. The number of projected fringes and the measurement volume size determine the number of expected periods mapped on the considered line segment $s$.

We assume the calibration including lens distortion determination (see $[14,16])$ being completed and the parameters projection center $\mathrm{O}=(\mathrm{X}, \mathrm{Y}, \mathrm{Z})$, orientation angles $\phi, \omega$, and $\kappa$ resulting in rotation matrix R, camera constant and principle point coordinates, and distortion description $\mathrm{D}$ of all components being known.

Ray $r$ is defined in the 3D space by the projection center, the orientation of camera $\mathrm{C}_{1}$, and point $p$. The predefined measurement volume $m v$ is assumed to be a convex polyhedron, say a rectangular solid in a meaningful position of the object space. The two intersection points of $r$ and $m v$ result in two 3D points $Q_{\min }$ and $Q_{\max }$ as shown in fig. 2. The image points $q_{\min }$ and $q_{\max }$ in the image of camera $\mathrm{C}_{2}$ are the undistorted segment endpoints of $s$. Hence applying distortion operator $\mathrm{D}_{2}$ (of camera $\mathrm{C}_{2}$ ) leads to the points $q^{\prime}{ }_{\text {min }}=\mathrm{D}_{2}\left(q_{\text {min }}\right)$ and $q^{\prime}{ }_{\text {max }}^{\prime}=\mathrm{D}_{2}\left(q_{\text {max }}\right)$ of segment $s^{\prime}$. For simplification in the following we assume no distortion being present (obtained e.g. by perfect correction, see $[14,16])$ and hence $s=s^{\prime}$.

In order to obtain $q_{\min }$ and $q_{\max }$ first the ray $r$ can be determined using $\mathrm{O}_{1}$ and $p$. Then we get $Q_{\min }$ and $Q_{\max }$ from $r$ and $m v$. Finally, we get $q_{\min }$ and $q_{\max }$ by applying the collinearity equations [14] using calibration data. For illustration see fig. 3.

The recording of the projected fringe sequence and calculation of the phase values leads to the distribution of the phase values on the epipolar segment $s$ (see fig. 2). If 
no occlusions are present, each projected fringe period provides one candidate to be the corresponding point $q$ to $p$.

Only in case of a very small measurement volume in direction of the optical axis or a very wide fringe width the number of mapped fringe periods on the epipolar segment is one and the point correspondence is unique. In general case a number of possible candidates $q_{i}$ having the same rough phase value $\phi_{p}$ correspond to $p$ leading to $n$ possible 3D point candidates $Q_{i}$ for the actual point $Q$.

However, the number of possible candidates corresponding to the number $N$ of projected fringe periods can be drastically reduced using further geometric properties of the sensor. Let us consider a back-projection of all 3D point candidates $q_{i}$ onto the projectors image plane leading to phase values $\phi_{i}$. If the point correspondence is true, the origin $\phi_{i}$ in the projector image plane of the point $q_{i}$ should correspond to the observed phase value $\phi_{p}: \phi_{i}-\phi_{p}=0$. If candidate $q_{i}$ is false, the measured phase value $\phi_{i}$ may differ from the expected one $\phi_{p}$. If the amount $\left|\delta_{i}\right|$ lof the difference $\delta_{i}=\phi_{i}-\phi_{p}$ is above a threshold $t h r$ candidate $q_{i}$ will be rejected (see fig. 3 ).

Rejection of false candidates is, unfortunately, not complete. However, the expected number of remaining false candidates can be estimated using statistical properties. Let us consider the general case of an arbitrarily shaped measuring object. The general measuring accuracy of the system using wrapped phases leads to a typical distribution of the $\phi_{d}$ values of the correct correspondences. Here the mechanic and thermic stability of the sensor, the quality of distortion correction, and other conditions influence the measuring accuracy. Assume we have a certain accuracy of the back-propagation phase measurement error, characterized by the standard deviation $s d$ of $\phi_{d}$. Phase values should be in the interval $[0,2 \pi]$.

Assuming a normal distribution of the $\delta$ values of correct correspondences, $99.9 \%$ have an absolute difference value of $\left|\delta_{q}\right|<3 s d$. Choosing as threshold thr $=3 s d$ and assuming a uniform distribution of the phase values of the false candidates. This means, that every false candidate has a probability of prob $=3 s d / \pi$ to be below the threshold $t h r$ of rejection. Multiplied with the average number $n$ of periods on one epipolar segment $s$ minus one it gives the expected number efc of additional false candidates per true candidate:

$$
e f c=3 s d(n-1) / \pi
$$

Using equation (1) we can initially estimate the expected number of remaining false candidates to be rejected in a subsequent step. The amount of efc indicates the probability of the algorithm described in the following to be successful. The lower the amount of $e f c$ the better the correspondence finding algorithm will work.

\subsection{Approach for Obtaining Unique Correspondences}

In this section the approach for the procedure of resolving ambiguities and obtaining unique point correspondences is described.

In a first step all candidates concerning the $\delta$ - criterion $\left(\left|\delta_{i}\right|<t h r\right)$ are collected. Each point in the $\mathrm{C}_{1}$ camera may have zero, one, or more corresponding candidates. In the first case there may be no correspondence because of hidden scene parts, image 
disturbances, or exceedance of the difference threshold $t h r$. These points have no relevance except leading to incomplete regions in the resulting 3D point cloud.

Those pairs of corresponding point candidates without additional candidate are true correspondence with high probability. However, there may be also false correspondences, namely in case of absence of the true corresponding point for point $p_{1}$.

In the third group of multiple corresponding candidates for one point $p_{1}$ the true correspondence is present in the set of candidates with high probability, except in the case described before.

Let an initial set of points in the image of camera $\mathrm{C}_{1}$ and a set of all initially found (still multiple) corresponding points in the image of $\mathrm{C}_{2}$ be given. A number of unique correspondences can be identified due to processing as explained above. To maximize the number of unique correspondences, the following steps are performed:

1. Selection of reference correspondences (assumed to be correct)

2. Selection of correspondences out of the set of points with multiple corresponding candidates

3. Decision whether uncertain correspondences are true or false

4. Rejection of correspondences by geometric contradictions (section 2.2.3)

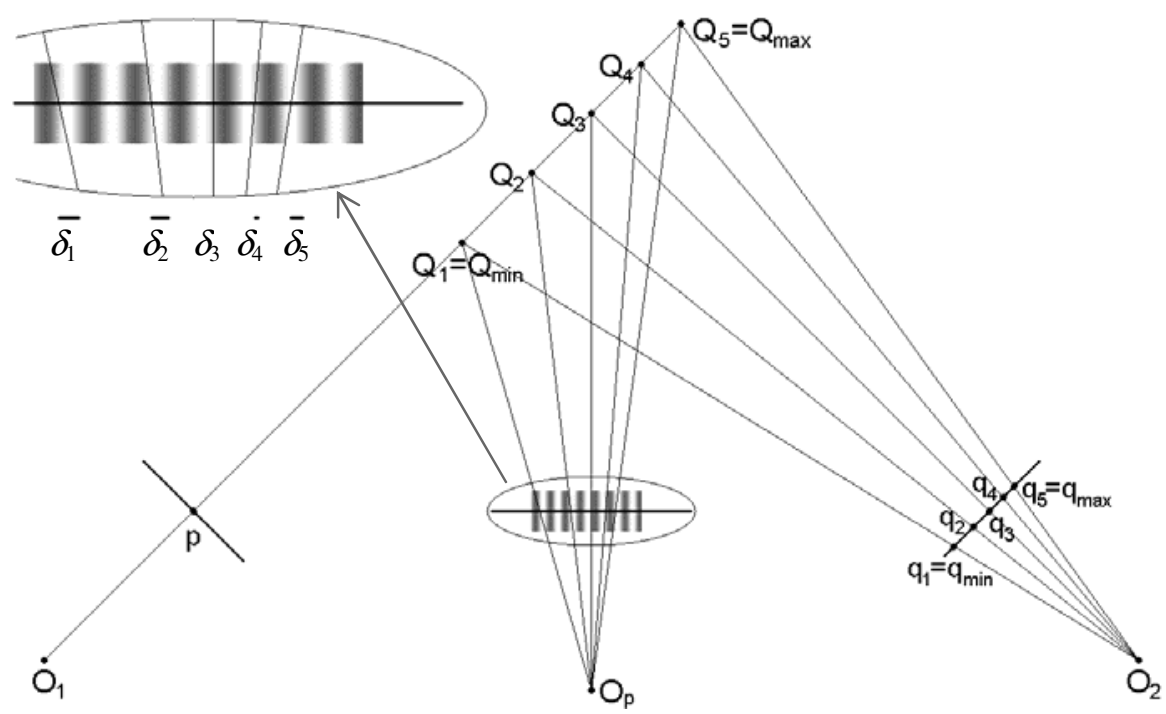

Fig. 3. Point $p$ and five candidates $q_{i}$ : three candidates $\left(q_{1}, q_{2}, q_{5}\right)$ are rejected because of too big difference values $\delta_{i}$, two candidates remain: correct one $\left(q_{3}\right)$ and false one $\left(q_{4}\right)$ - view from above. The phase values on projector image plane are represented by sinusoidal fringe pattern, the length of horizontal bars above the ' $\delta_{i}$ ' represents the amount of error $\delta_{i}$. Error $\delta_{3}$ is assumed to be zero.

\subsubsection{Selection of Reference Correspondences}

For the selection of reference correspondences (step 1) no fix algorithm may be given here, because the conditions of the measurement are different. However, some aspects should be noticed. First, an analysis of the expected number of false candidates 
according to equation (1) should be performed taking into account the expected accuracy of the projector phase and expansion of measurement volume. Additionally the expected number of unfound correspondences (occlusions, shadows, etc.) may be roughly estimated. The percentage of unique correspondences $p u c$ yields from equation (1) and is about $p u c \approx 100 \cdot(1-e f c)$ in the case of efc considerable smaller than one. The following properties are possible criteria for reference points.

- Exactly one corresponding candidate exists

- Neighbouring correspondences are similar (e.g. small difference in coordinates) and unique, too

- No violation of the monotony of the unwrapped phase and the corresponding coordinate along the epipiolar segment

\subsubsection{Selection of Correspondences of the Set of Points with Multiple Corresponding Candidates and Evaluation of Uncertain Points}

After the reference points are identified all remaining points are addressed. There are still uncertain candidates having exactly one correspondence and points with multiple corresponding candidates. Let the current candidate corresponding to point $p$ in the $\mathrm{C}_{2}$ image be $c(p)=q$. Consider a small surrounding of the corresponding epipolar segments $s_{1}$ and $s_{2}$ with $p$ and $q$ as center-points, respectively. The phase values at positions $p$ and $q$ are denoted by $\phi(p)$ and $\phi(q)$. Search for reference correspondences around $p$ and $q$ (see fig. 4) with maximal phase difference of $2 \pi$ with coordinates $k_{i}$, $g_{i}, c\left(k_{i}\right)$, and $c\left(g_{i}\right)$, respectively. If the correct corresponding point to $p$ is $q$, it must hold for phase values $\phi$ of all reference correspondence coordinates $c\left(k_{i}\right)$ and $c\left(g_{i}\right)$ :

$$
\begin{gathered}
\varphi\left(k_{i}\right)<\varphi(p) \wedge \varphi(p)-\varphi\left(k_{i}\right)<2 \pi \\
\varphi\left(g_{i}\right)>\varphi(p) \wedge \varphi\left(g_{i}\right)-\varphi(p)<2 \pi . \\
\varphi\left(c\left(k_{i}\right)\right)<\varphi(q) \forall k_{i} \\
\varphi\left(c\left(g_{i}\right)\right)>\varphi(q) \forall g_{i}
\end{gathered}
$$

If this holds for just one candidate, it must not be valid for all the other possible candidates. However, there may be contradictions for some candidates or no unique decision possible. In these cases correspondence must be fixed in a following step.
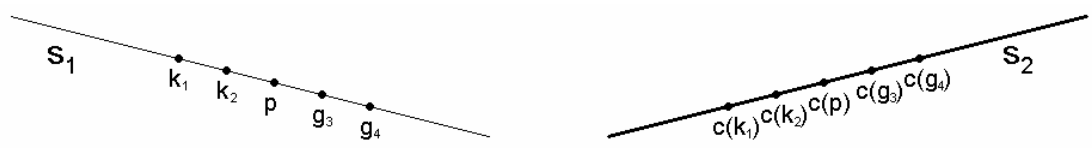

Fig. 4. Evaluation of uncertain point correspondence candidates $c(p)$ according to (2)

\subsubsection{Rejection of Found Correspondences by Contradictions and Additional Decisions}

All found correspondences may be tested using the feature of the monotony of the coordinates (only one coordinate is monotone!) and of the unwrapped phase values along the epipolar lines over the considered image areas. Contradictions may 
be found and monotony may be enforced. However, this should not be explicitly described here.

If the corresponding pairs of reference are not well distributed, which is, unfortunately the typical case, a number of correspondences remains open (more than one candidate is possible) or uncertain (current correspondence may be false). Decisions must be realized using other criteria. Here the regions are extended also perpendicular to the direction of the epipolar lines. The complete correspondence finding will be obtained by iterative application of the described procedure.

\subsection{Realization of the Method}

The method described so far was implemented in a test environment using measurement data from a measuring device at our institute. The results are given in the next section. Here the necessary preparation tasks are described.

First, initial analyzing experiments were performed including estimation of the back-propagation phase accuracy in order to set the threshold thr.

The implementation of the described algorithm was done by replacing the part of finding corresponding points in the current software package of our measuring system. As we assume well-known calibration values including the distortion function, the epipolar segments do not depend on the current measurement. Hence the corresponding segments to all image points $p$ of $\mathrm{C}_{1}$ can be determined before the measurement. The endpoints of the segments are stored in suitable data files. Point correspondence finding is performed for each point $p$ in the image of camera $\mathrm{C}_{1}$. The value of the rough phase $\phi_{p}$ is determined and the same phase values are searched for on segment $s$. All candidates $q_{i}$ with a small difference value $\left.\left(\mid \phi_{i}\right) \mid<t h r\right)$ become correspondence candidates. The selection of the true candidate is performed as described in the previous section.

\section{Experiments and Results}

The developed algorithm was implemented and tested using datasets obtained by the measuring device "kolibri Flex mini" [16]. This measuring system is a table-top system for measuring objects up to size of $80 \mathrm{~mm}$ (diameter) x $25 \mathrm{~mm}$ (height).

Rough phase data files were generated using unwrapped phase values obtained by a 16-phase algorithm (see [2]). The mean number $n$ of fringe periods per segment was $n=6$, and the mean standard deviation $s d$ of the back-propagation phase measurement error was $s d=0.041$ leading to an expected mean number of remaining false candidates of $e f c=0.20$ per correspondence.

In order to evaluate the developed method some experiments were performed. The goal was to get results in completeness, correctness, and accuracy. A number of different measuring objects were selected including a toy elephant, a prism, a single plaster tooth, a machine tool (see fig. 5), a set of teeth made of plaster, a plaster nose, and a plane surface. In order to compare the results to those of applying an algorithm including Gray code sequences a reference dataset (RD) was produced for every measurement including point correspondence data and 3D surface points. 

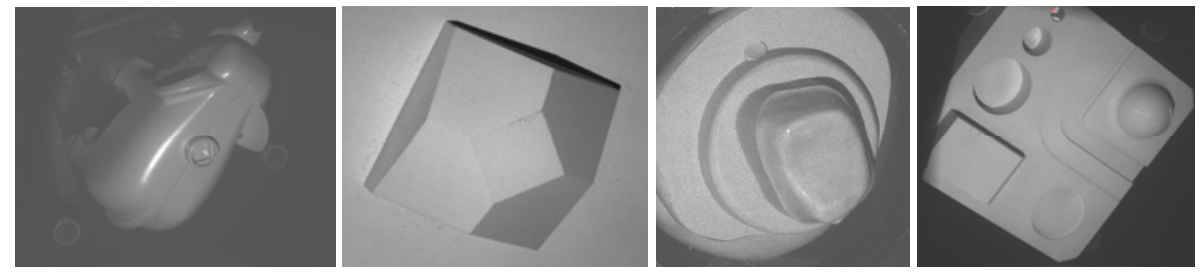

Fig. 5. Selected measuring objects: elephant, prism, plaster tooth, machine tool

The resulting point correspondences applying the new method were classified concerning the following characteristic features:

- Completeness com (percentage of the correctly found point correspondences concerning the number of point correspondences in RD)

- Completeness error miss (percentage of unfound correspondences)

- Correspondence error $f p c$ (percentage number of false positive point correspondences)

Because of the possibility of finding all correct point correspondences but having additionally some false positive point correspondences, the sum of com + miss $+f p c$ may exceed $100 \%$. Additionally, found point correspondences were used to produce 3D measurement data. These datasets were used to compare the measurement results to the reference results. The results for the characteristic features com, miss, and $f p c$ are presented in table 1 . The higher error of the nose object is among others (hidden parts) due to extended height (about $45 \mathrm{~mm}$ ) of the object leading to higher efc value.

Sequence length was significantly reduced according to the method used so far (see table 2). Some reconstructed object surfaces obtained by processing of the 3D point clouds are shown in fig. 6.

Table 1. Completeness Results

\begin{tabular}{lcccr}
\hline Object & com in $\%$ & miss in $\%$ & $f p c$ in $\%$ & number of points \\
\hline Plane & 100.00 & 0.00 & 0.00 & 6384 \\
Prism & 99.96 & 0.03 & 0.02 & 19307 \\
Machine tool & 99.99 & 0.01 & 0.03 & 28568 \\
Set of teeth & 99.99 & 0.01 & 0.03 & 70249 \\
Plaster tooth & 100.00 & 0.00 & 0.13 & 6769 \\
Nose & 99.92 & 0.06 & 2.02 & 22709 \\
Elephant & 99.99 & 0.01 & 0.00 & 11412 \\
\hline
\end{tabular}
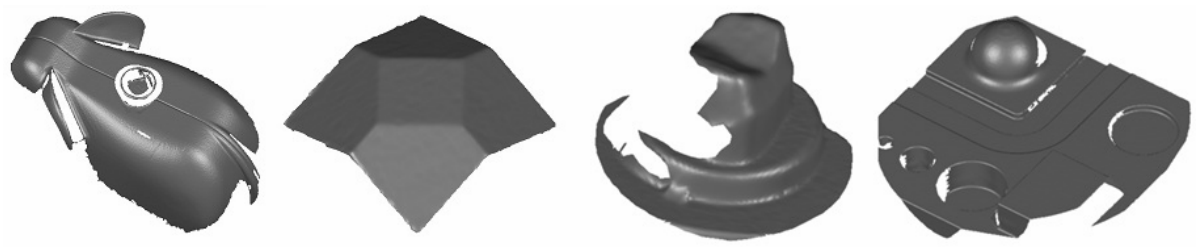

Fig. 6. Results (3D surfaces) of selected measuring objects 
Table 2. Sequence length (number of images) according to several phase algorithms [2] ( $\mathrm{GC}=7$ image Gray code), PA achieves reduction to $70 \%, 54 \%$, and $37 \%$ concerning EGC

\begin{tabular}{llll}
\hline Algorithm & 16-phase & 8-phase & 4-phase \\
\hline Conventional, two directions, full GC (CGC) & 46 & 30 & 22 \\
Full GC with epipolar lines (EGC) & 23 & 15 & 11 \\
Proposed algorithm without GC (PA) & 16 & 8 & 4 \\
\hline
\end{tabular}

\section{Summary, Discussion, and Outlook}

We introduced a new method for fringe projection based 3D stereo scanners is introduced which realizes point correspondence finding and subsequent unwrapping of phase images without binary codes. It works robust if certain geometric properties hold. The geometric constraints of all optical sensor components were used in combination of the expected measuring accuracy of the sensor. A considerable reduction of the projected fringe code can be achieved by omission of the entire Gray code sequence resulting in a faster image sequence recording. This is important for high speed applications, e.g. in handheld 3D measurement systems.

It could be shown experimentally, that a completeness of $99.9 \%$ and an error rate of about $0.1 \%$ in the case of $e f c=0.2$ and $2.0 \%$ at $e f c=0.5$ can be achieved depending also on the object properties. This is sufficient for practical applications because the 3D points resulting from false positive point correspondences may be eliminated in the 3D space by suitable outlier detection algorithms. However, we assume a worse performance of the algorithm at values of efc greater than about 1.0 corresponding to an extended measuring volume depth. We recommend applying the method in the case of $e f c$ smaller than about 0.5 . Alternatively, fringe period length can be extended or, if possible, back-propagation phase error must be reduced by some algorithmic or hardware improvement.

The image recording time can be considerably reduced compared to conventional image recording using Gray code and providing the same accuracy of the measurement. This leads to significant saving of measurement time. On the other hand, the point correspondence finding algorithm as described needs more computational effort than using Gray code sequences. Because the algorithm needs to be applied iteratively, the computation time may considerably vary. Hence an estimation of additional computation time is difficult.

However, computation time in average and worst case, respectively, will be analysed in a next step. Furthermore, calculation time of the algorithm has to be optimized. Additionally, future work should also include implementation of the algorithm into several measuring systems for 3D surface measurement using fringe projection and performing experiments using other sensor systems in order to evaluate the algorithm.

\section{References}

1. Battle, J., Mouaddib, E., Salvi, J.: Recent progress in coded structured light as a technique to solve the correspondence problem: a survey. PR 31(7), 963-982 (1998)

2. Li, E.B., Peng, X., Xi, J., Chicaro, J.F., Yao, J.Q., Zhang, D.W.: Multi-frequency and multiple phase-shift sinusoidal fringe projection for 3D profilometry. Optics Express 13, 1561-1569 (2005) 
3. Zhang, H., Lalor, M.J., Burton, D.R.: Spatiotemporal phase unwrapping for the measurement of discontinuous objects in dynamic fringe-projection phase-shifting profilometry. Applied Optics 38, 3534-3541 (1999)

4. Sansoni, G., Carocci, M., Rodella, R.: Three-dimensional vision based on a combination of Gray-code and phase-shift light projection: Analysis and compensation of the systematic errors. Applied Optics 38(31), 6565-6573 (1999)

5. Zhang, S., Yau, S.T.: High-resolution, real-time 3D absolute coordinate measurement based on a phase-shifting method. Optics Express 14(7), 2644-2649 (2006)

6. Ishiyama, R., Sakamotom, S., Tajima, J., Okatani, T., Deguchi, K.: Absolute phase measurements using geometric constraints between multiple cameras and projectors. Applied Optics 46(17), 3528-3538 (2007)

7. Ishiyama, R., Okatani, T., Deguchi, K.: Precise 3-d measurement using uncalibrated pattern projection. In: Proc. IEEE Int. Conf. on Image Proc., vol. 1, pp. 225-228 (2007)

8. Young, M., Beeson, E., Davis, J., Rusinkiewicz, S., Ramamoorthi, R.: Viewpoint-coded structured light. In: Proc. CVPR, pp. 1-8 (2007)

9. Li, Z., Shi, Y., Wang, C.: Real-time complex object 3D measurement. In: Proc. ICCMS, pp. 191-194 (2009)

10. Bräuer-Burchardt, C., Munkelt, C., Heinze, M., Kühmstedt, P., Notni, G.: Phase unwrapping in fringe projection systems using epipolar geometry. In: Blanc-Talon, J., Bourennane, S., Philips, W., Popescu, D., Scheunders, P. (eds.) ACIVS 2008. LNCS, vol. 5259, pp. 422-432. Springer, Heidelberg (2008)

11. Bräuer-Burchardt, C., Munkelt, C., Heinze, M., Kühmstedt, P., Notni, G.: Fringe code reduction for 3D measurement systems using epipolar geometry. In: Proc. PCVIA, ISPRS, vol. XXXVIII, Part 3A, pp. 192-197 (2010)

12. Kühmstedt, P., Heinze, M., Himmelreich, M., Bräuer-Burchardt, C., Notni, G.: Optical 3D sensor for large objects in industrial application. In: Proc. SPIE, vol. 5856, pp. 118-127 (2005)

13. Kühmstedt, P., Munkelt, C., Heinze, M., Bräuer-Burchardt, C., Notni, G.: 3D shape measurement with phase correlation based fringe projection. In: Proc. SPIE, vol. 6616, 66160B (2007)

14. Luhmann, T., Robson, S., Kyle, S., Harley, I.: Close range photogrammetry. Wiley Whittles Publishing, Chichester (2006)

15. Schreiber, W., Notni, G.: Theory and arrangements of self-calibrating whole-body threedimensional measurement systems using fringe projection technique. Opt. Eng. 39, 159 $169(2000)$

16. Bräuer-Burchardt, C., Heinze, M., Munkelt, C., Kühmstedt, P., Notni, G.: Distance dependent lens distortion variation in 3D measuring systems using fringe projection. In: Proc. 17th BMVC, pp. 327-336 (2006) 